\title{
SOLVENT FREE OXALIC ACID CATALYZED SYNTHESIS OF 1,5-BENZODIAZEPINES
}

\author{
ANIKET P. SARKATE, ${ }^{a} J A I P R A K A S H ~ N . ~ S A N G S H E T T I,{ }^{b}$ NANASAHEB B. DHARBALE, ${ }^{c}$ AJINKYA P. SARKATE, ${ }^{d}$ \\ PRAVIN S. WAKTE, ${ }^{a}$ DEVANAND B. SHINDE ${ }^{a *}$
}

\author{
${ }^{a}$ Department of Chemical Technology, Dr. Babasaheb Ambedkar Marathwada University, Aurangabad 431004, MS, India. \\ ${ }^{b}$ Department of Pharmaceutical Chemistry, Y. B. Chavan College of Pharmacy, Aurangabad 431003 (MS), India. \\ 'Department of Pharmaceutical Chemistry, Shri Bhagwan College of Pharmacy, Aurangabad 431003 (MS), India. \\ ${ }^{d}$ Department of Pharmaceutical Chemistry, Sinhgad Institute of Pharmacy, Pune 411041 (MS), India
}

(Received: January 8, 2013 - Accepted: April 12, 2013)

\begin{abstract}
In the present study 1, 5-benzodiazepines were synthesized from a range of $\alpha, \beta$-unsaturated ketones and $o$-phenylendiamine using oxalic acid 10 mol\% as a catalyst under solvent free conditions. The yields of the present method are better than the reported method which explains effectiveness of oxalic acid catalyst. The cost effective, resourceful, undemanding and environment friendly are the advantageous aspects of this method.
\end{abstract}

Keywords: 1, 5-benzodiazepine, oxalic acid, solvent free conditions, $\alpha, \beta$-unsaturated ketones, o-phenylenediamine.

\section{INTRODUCTION}

Benzodiazepines are important class of heterocyclic scaffolds because of their commercial importance and clinical success. The pharmacological activities of benzodiazepines ${ }^{1}$ like anxiolytics, hypnotics, tranquilizers, and anticonvulsants, have led to their recognition by medicinal community as the structure of particular significance. ${ }^{2}$ Although the first benzodiazepine was introduced as a drug nearly 40 years ago, ${ }^{3}$ the research in this area is still very active in view of producing the compounds of enhanced pharmacological activity. In the last decade, the area of biological interest of 1, 5-benzodiazepines has been extended to several diseases such as cancer, viral infection, and cardiovascular disorders. ${ }^{4}$ Moreover 1, 5-benzodiazepines are key intermediates for the synthesis of various fused ring systems such as triazolo-, oxadiazolo-, oxazino- or furanobenzodiazepines. ${ }^{5}$ In addition, benzodiazepine derivatives also have commercial importance as dyes for acrylic fibres in photography. ${ }^{6}$

Most of the methods reported in the literature for the synthesis of benzodiazepines either includes (i) condensation of $o$-phenylenediamine with $\alpha, \beta$-unsaturated ketones ${ }^{7}$ or (ii) the reaction of $o$-phenylenediamines with various ketones using variety of reagents and catalysts. These includes, (i) $\mathrm{BF}_{3}$ $\mathrm{Et}_{2} \mathrm{O},{ }^{8} \mathrm{NaBH}_{4},{ }^{9}$ polyphosporic acid, ${ }^{10}$ ceric ammonium nitrate $(\mathrm{CAN}),{ }^{11}$ and $\mathrm{TCT}^{12}$ in the presence of various solvents; (ii) $\mathrm{MgO}$ and $\mathrm{POCl}_{3},{ }^{13} \mathrm{Yb}(\mathrm{OTf})_{3},{ }^{14}$ $\mathrm{Hg}(\mathrm{OTf})_{3},{ }^{15} \mathrm{CeCl}_{3} 7 \mathrm{H}_{2} \mathrm{O} / \mathrm{NaI}$ supported on silica gel, ${ }^{16} \mathrm{Sc}(\mathrm{OTf})_{3},{ }^{17} \mathrm{Er}(\mathrm{OTf})_{3}$, $\mathrm{InBr}_{3}{ }^{18}{ }^{1}$ sulphated zirconia, ${ }^{19} \mathrm{ZnCl}_{2}{ }^{20}$ and $\mathrm{SiO}_{2} / \mathrm{ZnCl}_{2}{ }^{21}$ under solvent free conditions; (iii) $\mathrm{Al}_{2} \mathrm{O}_{3} / \mathrm{P}_{2} \mathrm{O}_{5}^{22}$ or acetic acid ${ }^{23}$ with microwave irradiation; (iv) Amberlyst-15 in ionic liquids; ${ }^{24}$ (v) 1,3-di- $n$-butylimidazolium bromide without any catalyst. ${ }^{25}$ Literature survey also reveals the method of Shrinivasan and co-workers, a multicomponent reaction for the synthesis of 3-Hbenzo[b]-1,4diazepines. ${ }^{26}$ Recently, Jiang and co-workers reveals $[4+2+1]$ cycloaddition of $o$-phenylenediamine and ethyl propionate to synthesize 3, 4-disubstituted 1,5 -benzodiazepines in good yield. ${ }^{27}$

Oxalic acid is the compound in which two carboxylic groups are joined together directly and hence supposed to be one of the strongest organic acid. The use of oxalic acid as a catalyst was reported for the deprotection of ketal to give the corresponding aldehydes or ketones. ${ }^{28}$ It was also found to be used for isomerisation of $\delta^{5}$-cholasten-3-one to $\delta^{4}$-cholesten-3-one ${ }^{29}$ and dealumination of zeolite. ${ }^{30}$ Furthermore, we have used oxalic acid as a catalyst in our previous study for the synthesis of dihydropyrimidine 2-(1H)-ones, ${ }^{31}$ 2 -aryl-1-arylmethyl-1 $H$-benzimidazoles and 2,4,5-triaryl- $1 H$-imidazoles, ${ }^{32}$ quinazolin-4(3H)-ones ${ }^{33}$ and coumarins. ${ }^{34}$ In order to explore the use of oxalic acid catalyst further, herein we report the synthesis of 1, 5-benzodiazepines from $o$-phenylene diamine and various $\alpha, \beta$-unsaturated ketones.

\section{RESULT AND DISCUSSION}

Although development of nonhazardous synthetic methodologies for organic reactions is one latest challenge to the organic chemists, only few of the benzodiazepine synthesis methods tried to answer this demand. Nevertheless, the search for new methodology to synthesize benzodiazepine and analogs continues.
In an attempt to identify the most optimal experimental conditions a detailed study was performed on $o$-phenylenediamine (4) and $\alpha, \beta$-unsaturated ketones (3a) (chalcone) (Scheme 2). The reaction condition has been optimized with respect to catalyst loading and use of solvents (Table1). Synthesis of target molecule has been tried using different solvents like DMF, 1, 4-dioxane, THF, etc. Different solvents promote the formation of target compounds with low to moderate yields. The reaction has also been carried out in aqueous conditions where the reaction found to be very sluggish and very trace amount of product has been formed. From the result it is observed that use of $10 \mathrm{~mol} \%$ catalyst is more favourable, giving the product with $93 \%$ yield. However, only modest yield of condensation product was detected at room temperature, probably due to low nucleophilicity of the amino group, as described in previous reports. ${ }^{35}$ But, good results were registered for the formation of 1,5 benzodiazepine $\mathbf{5 a - j}$ almost in quantitative yield ranging from $72-88 \%$ when the reaction was carried out at about $80^{\circ} \mathrm{C}$ temperature. The highest yield of $88 \%$ was registered with ACN whilst, only traces of the product formed when the reaction was carried out in water. Furthermore, the study was also carried out in nonpolar solvents like DCM and chloroform (Table 1, entries 6 and 7), but the yields registered were less with added reaction time at $80^{\circ} \mathrm{C}$ and no product was detected at room temperature.

Taking into consideration the green synthetic approach and hazards caused by organic solvents the reaction was further tried under solvent free conditions. Surprisingly, the product formed was registered with $91 \%$ yield with $20 \mathrm{~mol} \%$ of the catalyst and $93 \%$ with $10 \mathrm{~mol} \%$ (Table 1 , entries 8 and 9 respectively). Further reduction in mol\% of the catalyst resulted in lower yields and added reaction time (Table 1, entries 10 and 11).

Therefore, it was concluded that $10 \mathrm{~mol} \%$ of oxalic acid catalyst was sufficient to form 1, 5-benzodiazepine in almost quantitative yield of the isolated product under solvent free conditions. Hence, the same reaction conditions were followed for the further synthesis of 1, 5-benzodiazepine analogs 5a-j (Table 2).

In an attempt to study the effect of substituted chalcones in the condensation, we have carried out the condensation of o-phenylenediamine (4) with these substituted chalcones (3a-j) (Scheme 2). The best results for the condensation were registered for the benzodiazepines substituted with electron releasing groups like $-\mathrm{OH},-\mathrm{OMe},-\mathrm{CH}_{3}$ at ortho and para positions, however highest yield was registered with benzodiazepine with the $-\mathrm{OH}$ group at ortho position and -OMe group at para position (Table 2, Entry $\mathbf{5} \mathbf{j}$ and $\mathbf{5 h}$ respectively). In addition to this the reaction was also tried for electron withdrawing substituents which resulted in the the products with moderate yield (Table 2, Entry $\mathbf{5 b}, \mathbf{5 d}$ and $\mathbf{5 f}$ ).

Therefore, we can conclude that we have demonstrated the method to study an efficiency of oxalic acid catalyst by synthesizing an assorted 1,5-benzodiazepines (Table 2, Entry $\mathbf{5 a - j}$ ) using $\mathbf{4}$ and $\mathbf{3 a - j}$ in solvent free conditions. Further, we have also demonstrated observation regarding the effect of different substituents on the quantitative yield of isolated product. Moreover, easy work up, shorter reaction time, good to better yields and environment friendly were the advantages of the anticipated method. 


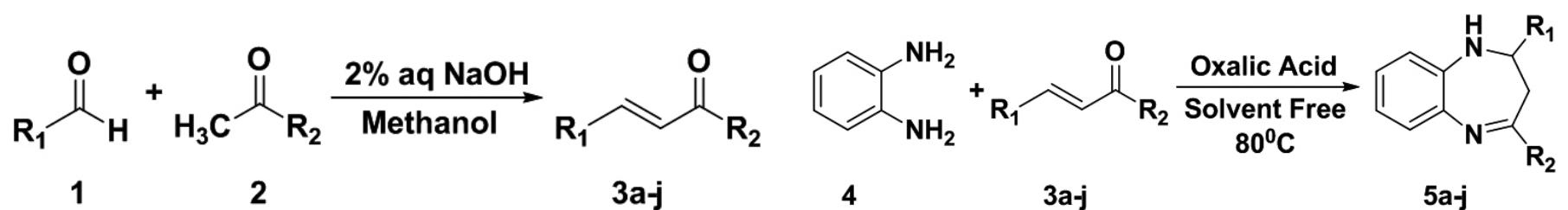

Scheme 1 Synthesis of $\alpha, \beta$-unsaturated ketones 3a-j from substituted aldehydes and ketones. 3a-j

Scheme 2 Scheme for the synthesis of 1, 5-benzodiazepines (5a-j) from

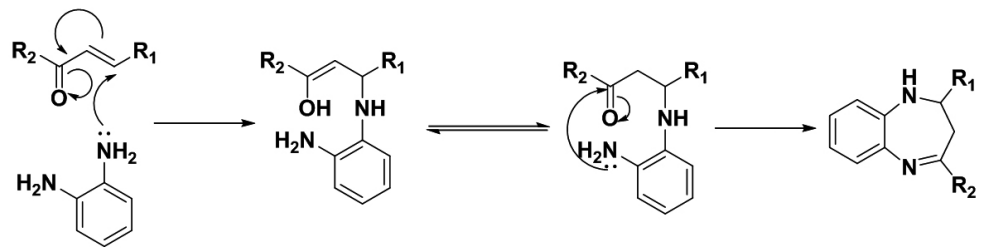

Scheme 3 Proposed mechanism for the synthesis of benzodiazepine

Table 1 Optimization of reaction conditions and mol\% of oxalic acid for the synthesis of $\mathbf{5 a}$ (Entry 1, Table 2).

\begin{tabular}{|c|c|c|c|c|}
\hline Entry & Solvent & Oxalic acid (mol\%) & Reaction time (min) & Yield (\%) \\
\hline 1 & DMF & 20 & 180 & 84 \\
\hline 2 & $1,4-$ dioxane & 20 & 160 & 72 \\
\hline 3 & THF & 20 & 150 & 76 \\
\hline 4 & $\mathrm{ACN}$ & 20 & 155 & 88 \\
\hline 5 & $\mathrm{H}_{2} \mathrm{O}$ & 180 & Trace \\
\hline 6 & $\mathrm{DCM}$ & 20 & 175 & 62 \\
\hline 7 & $\mathrm{CHCl}_{3}$ & 20 & 185 & 54 \\
\hline 8 & Solvent free & 20 & 135 & 91 \\
\hline 9 & Solvent free & 20 & 150 & 93 \\
\hline 10 & Solvent free & 10 & 185 & 80 \\
\hline 11 & Solvent free & 5 & 210 & \\
\hline
\end{tabular}

${ }^{a}$ yields are of pure isolated compound

\section{EXPERIMENTAL}

All reagents and chemicals were of analytical grade and used without further purification.

Melting points of all the compounds were determined using digital melting point apparatus (SRS) and were uncorrected. IR spectrum of all compounds was recorded with FT-IR spectrophotometer (Brucker). ${ }^{1}$ HNMR (Varian NMR $400 \mathrm{~Hz}$ ) of the compound $\mathbf{5 a - j}$ was recorded in $\mathrm{CDCl}_{3}$ using TMS as an internal standard and molecular weight was confirmed by using LC-MS (Scinpor ESI)

3.1 General procedure for the synthesis of $3 \mathrm{a}-\mathrm{j}$.

In a round bottom flask, $2.2 \mathrm{~g}$ of sodium hydroxide was added in water \& methanol. The flask was immersed in a bath of crushed ice \& kept on mechanical stirrer. The stirrer was started, freshly distilled acetophenone $(0.43$ mol) \& pure benzaldehyde $(0.43 \mathrm{~mol})$ was added in the flask. The mixture was stirred for $4-5 \mathrm{~h}$ at $15-20^{\circ} \mathrm{C}$. The thick mixture was removed \& kept in a refrigerator for overnight. The product was filtered \& washed with cold water until the washings were neutral to litmus. Further the product was washed with ice cold rectified spirit, dried \& recrystallized from rectified spirit to afford the desired product.

3.2 General procedure for the synthesis of $5 \mathrm{a}-\mathrm{j}$ (5a)

3.2.1 Synthesis of 2,3-dihydro-2,4-diphenyl-1H-benzo[b][1,5]diazepine

A mixture of $\alpha, \beta$-unsaturated ketones 3a (10 mmol), $o$-phenylenediamine $4(10 \mathrm{mmol})$ and oxalic acid $(10 \mathrm{~mol} \%)$ was heated at $80^{\circ} \mathrm{C}$ under solvent free conditions for the appropriate time about $150 \mathrm{~min}$. After completion of reaction (monitored by TLC), the reaction mixture was cooled at room temperature and extracted with diethyl ether $(3 \times 10 \mathrm{ml})$. The combined organic layers were dried over anhydrous $\mathrm{Na}_{2} \mathrm{SO}_{4}$. The solvent was removed and the solid residue was purified further on silica gel column $(2.5 \times 45 \mathrm{~cm})$, pre packed in chloroform. Elution of the column with petroleum ether:ethyl acetate (18:2) gave a homogeneous residue which was concentrated in vacuo to give compound $\mathbf{5 a}$. Yield: $96 \%$, MP (SRS melting point apparatus): $136-137^{\circ} \mathrm{C}$; ${ }^{1} \mathrm{H}$ NMR (400
$\mathrm{MHz}, \mathrm{CDCl}_{3}, \delta$ ppm) $\delta: 1.9\left(\mathrm{~d}, 2 \mathrm{H}, \mathrm{CH}_{2}\right), 2.2(\mathrm{t}, 1 \mathrm{H}, \mathrm{CH}), 3.9(\mathrm{~s}, 1 \mathrm{H}, \mathrm{NH})$, 6.9-7.2 (8H, Ar-H), 7.4-7.8 (4H, Ar-H), 7.8-8 (2H, Ar-H); ${ }^{13} \mathrm{C} \mathrm{NMR}\left(\mathrm{CDCl}_{3}\right)$ $\delta=38.2,63.4,113.6,122.4,125.9,126.2,127.3,127.8,128.1,128.9,129.7$, 132.4, 139.9, 142.3, 144.8, 146.4, 163.3; IR (KBr): $1592(\mathrm{C}=\mathrm{C}), 1643(\mathrm{C}=\mathrm{N})$ $\mathrm{cm}^{-1}$; LC-MS (Scinpor ESI m/z): $298.38\left(\mathrm{M}^{+}\right), 299.25(\mathrm{M}+1)$; MF: $\mathrm{C}_{21} \mathrm{H}_{18} \mathrm{~N}_{2}$.

3.2.2 Compound (5b): 2-(4-chlorophenyl)-2,3-dihydro-4-phenyl-1Hbenzo[b][1,5]diazepine

Yield: $87 \%$, MP: $110-112^{\circ} \mathrm{C}$; ${ }^{1} \mathrm{HNMR}\left(400 \mathrm{MHz}, \mathrm{CDCl}_{3}, \delta \mathrm{ppm}\right): \delta 1.7(\mathrm{~d}$, $\left.2 \mathrm{H}, \mathrm{CH}_{2}\right), 2.9(\mathrm{t}, 1 \mathrm{H}, \mathrm{CH}), 4.2(\mathrm{~s}, 1 \mathrm{H}, \mathrm{NH}), 7.18-7.16(4 \mathrm{H}, \mathrm{Ar}-\mathrm{H}), 7.23-7.64$ $(5 \mathrm{H}, \mathrm{Ar}-\mathrm{H}), 6.8-7.2(2 \mathrm{H}, \mathrm{Ar}-\mathrm{H}) ;{ }^{13} \mathrm{C} \mathrm{NMR}\left(\mathrm{CDCl}_{3}\right) \delta=38.6,62.1,115.4,120.7$, $122.2,125.7,127.4,127.9,128.8,129.5,130.6,133.1,137.4,142.2,143.6$, 146.3, 161.8; IR (KBr): $1570(\mathrm{C}=\mathrm{C}), 1638(\mathrm{C}=\mathrm{N}) \mathrm{cm}^{-1}$; LC-MS (Scinpor ESI m/z): $332.69\left(\mathrm{M}^{+}\right), 334.09(\mathrm{M}+2,33.9 \%), 333.71(\mathrm{M}+1,22.8 \%)$; MF: $\mathrm{C}_{21} \mathrm{H}_{17} \mathrm{ClN}_{2}$.

3.2.3 Compound (5c): Synthesis of 2-(2,3-dihydro-2-(3,4,5trimethoxyphenyl)-1H-benzo[b] [1,5]diazepin-4-yl)-5-methylphenol

Yield: 89\%, MP: $96-98^{\circ} \mathrm{C} ;{ }^{1} \mathrm{HNMR}\left(400 \mathrm{MHz}, \mathrm{CDCl}_{3}, \delta \mathrm{ppm}\right): \delta 1.83(\mathrm{~d}$, $\left.2 \mathrm{H}, \mathrm{CH}_{2}\right), 2.73(\mathrm{t}, 1 \mathrm{H}, \mathrm{CH}), 3.85(\mathrm{~s}, 1 \mathrm{H}, \mathrm{NH}), 6.18(2 \mathrm{H}, \mathrm{Ar}-\mathrm{H}), 6.65-7.2(3 \mathrm{H}$, Ar-H), 6.42-7.11 (4H, Ar-H), $4.85(\mathrm{~s}, 1 \mathrm{H}, \mathrm{OH}), 2.15\left(\mathrm{~s}, 3 \mathrm{H}, \mathrm{CH}_{3}\right), 3.35(\mathrm{~s}, 9 \mathrm{H}$, $\left.\mathrm{OCH}_{3}\right) ;{ }^{13} \mathrm{C} \mathrm{NMR}\left(\mathrm{CDCl}_{3}\right) \delta=20.9,38.7,55.3,62.1,64.9,101.2,113.5,117.2$, $118.8,122.3,124.4,125.7,126.4,129.6,136.6,139.1,140.1,143.4,146.6$, 154.1, 159.9, 164.1; IR (KBr): $3417(\mathrm{O}-\mathrm{H}$, broad $), 1543(\mathrm{C}=\mathrm{C}), 1642(\mathrm{C}=\mathrm{N})$ $\mathrm{cm}^{-1}$; LC-MS (Scinpor ESI m/z): $418.10\left(\mathrm{M}^{+}\right), 419.45(\mathrm{M}+1,27.34 \%)$; MF: $\mathrm{C}_{25} \mathrm{H}_{26} \mathrm{~N}_{2} \mathrm{O}_{4}$.

3.2.4 Compound (5d): Synthesis of 2-bromo-6-(2,3-dihydro-2-(3,4,5trimethoxyphenyl)-1H-benzo[b][1,5]diazepin-4-yl)-4-methylphenol

Yield: 76\%, MP: $113-114^{\circ} \mathrm{C}$; ${ }^{1} \mathrm{HNMR}\left(400 \mathrm{MHz}, \mathrm{CDCl}_{3}, \delta \mathrm{ppm}\right): \delta 1.56$ $\left(\mathrm{d}, 2 \mathrm{H}, \mathrm{CH}_{2}\right), 3.12(\mathrm{t}, 1 \mathrm{H}, \mathrm{CH}), 4.01(\mathrm{~s}, 1 \mathrm{H}, \mathrm{NH}), 6.22(2 \mathrm{H}, \mathrm{Ar}-\mathrm{H}), 7.07-7.19$ $(2 \mathrm{H}, \mathrm{Ar}-\mathrm{H}), 6.37-7.01(4 \mathrm{H}, \mathrm{Ar}-\mathrm{H}), 4.54(\mathrm{~s}, 1 \mathrm{H}, \mathrm{OH}), 2.35\left(\mathrm{~s}, 3 \mathrm{H}, \mathrm{CH}_{3}\right), 3.42$ $\left(\mathrm{s}, 9 \mathrm{H}, \mathrm{OCH}_{3}\right) ;{ }^{13} \mathrm{C} \mathrm{NMR}\left(\mathrm{CDCl}_{3}\right) \delta=19.3,40.1,57.3,62.1,64.4,101.3,113.9$, $116.6,119.7,123.1,125.2,126.9,128.1,134.1,136.2,137.1,138.9,142.6$, 146.7, 151.2, 159.3, 163.9; IR (KBr): $3387(\mathrm{O}-\mathrm{H}$, broad), $1585(\mathrm{C}=\mathrm{C}), 1641$ 
$(\mathrm{C}=\mathrm{N}) \mathrm{cm}^{-1}$; LC-MS (Scinpor ESI m/z): $497.10\left(\mathrm{M}^{+}\right), 498.45(\mathrm{M}+1,98.34 \%)$; MF: $\mathrm{C}_{25} \mathrm{H}_{25} \mathrm{BrN}_{2} \mathrm{O}_{4}$

3.2.5 Compound (5e): Synthesis of 4-(2,3-dihydro-2-phenyl-1H-benzo[b] [1,5]diazepin-4-yl) phenol

Yield: $91 \%$, MP: $122-123^{\circ} \mathrm{C}$; ${ }^{1} \mathrm{HNMR}\left(400 \mathrm{MHz}, \mathrm{CDCl}_{3}, \delta \mathrm{ppm}\right): \delta 2.01$ (d, $\left.2 \mathrm{H}, \mathrm{CH}_{2}\right), 2.98$ (t, 1H, CH), $4.11(\mathrm{~s}, 1 \mathrm{H}, \mathrm{NH}), 7.08-7.27(5 \mathrm{H}, \mathrm{Ar}-\mathrm{H}), 6.45-$ $7.35(4 \mathrm{H}, \mathrm{Ar}-\mathrm{H}), 6.3-7.11(4 \mathrm{H}, \mathrm{Ar}-\mathrm{H}), 4.87(\mathrm{~s}, 1 \mathrm{H}, \mathrm{OH}) ;{ }^{13} \mathrm{C} \mathrm{NMR}\left(\mathrm{CDCl}_{3}\right)$ $\delta=20.2,40.1,64.3,113.3,117.4,120.2,124.1,125.4,126.9,127.6,130.8$, 135.2, 138.3, 142.9, 143.8, 146.2, 159.5, 164.3; IR (KBr): 3413 (O-H, broad), $1592(\mathrm{C}=\mathrm{C}), 1636(\mathrm{C}=\mathrm{N}) \mathrm{cm}^{-1}$; LC-MS (Scinpor ESI m/z): $314.10\left(\mathrm{M}^{+}\right)$; MF: $\mathrm{C}_{21} \mathrm{H}_{18} \mathrm{~N}_{2} \mathrm{O}$.

3.2.6 Compound (5f): Synthesis of 2,3-dihydro-2,4-bis(4-nitrophenyl)1H-benzo[b][1,5] diazepine

Yield: $82 \%$, MP: $131-132^{\circ} \mathrm{C}$; ${ }^{1} \mathrm{HNMR}\left(400 \mathrm{MHz}, \mathrm{CDCl}_{3}, \delta \mathrm{ppm}\right.$ ): $\delta 1.85$ (d, $\left.2 \mathrm{H}, \mathrm{CH}_{2}\right), 2.76(\mathrm{t}, 1 \mathrm{H}, \mathrm{CH}), 3.96(\mathrm{~s}, 1 \mathrm{H}, \mathrm{NH}), 7.21-8.15(4 \mathrm{H}, \mathrm{Ar}-\mathrm{H}), 7.7-$ $8.35(4 \mathrm{H}, \mathrm{Ar}-\mathrm{H}), 6.32-7.07(4 \mathrm{H}, \mathrm{Ar}-\mathrm{H}) ;{ }^{13} \mathrm{C}$ NMR $\left(\mathrm{CDCl}_{3}\right) \delta=37.9,62.1$, $113.2,120.5,122.3,124.4,125.1,126.2,127.4,129.2,140.3,142.7,144.2$, 145.3, 147.9, 149.1, 163.9; IR (KBr): 1351, $1555\left(\mathrm{NO}_{2}\right), 1577$ (C=C), 1648 $(\mathrm{C}=\mathrm{N}) \mathrm{cm}^{-1}$; LC-MS (Scinpor ESI m/z): $388.74\left(\mathrm{M}^{+}\right), 389.23(\mathrm{M}+1,23.33 \%)$ MF: $\mathrm{C}_{21} \mathrm{H}_{16} \mathrm{~N}_{4} \mathrm{O}_{4}$.

3.2.7 Compound (5g): Synthesis of 2,3-dihydro-2,4-dip-tolyl-1H-benzo[b] $[1,5]$ diazepine

Yield: $92 \%$, MP: $130-132^{\circ} \mathrm{C} ;{ }^{1} \mathrm{HNMR}\left(400 \mathrm{MHz}, \mathrm{CDCl}_{3}, \delta \mathrm{ppm}\right): \delta 2.11$ (d, $\left.2 \mathrm{H}, \mathrm{CH}_{2}\right), 2.99(\mathrm{t}, 1 \mathrm{H}, \mathrm{CH}), 4.22(\mathrm{~s}, 1 \mathrm{H}, \mathrm{NH}), 6.98-7.15(4 \mathrm{H}, \mathrm{Ar}-\mathrm{H}), 7.09-$ $7.54(4 \mathrm{H}, \mathrm{Ar}-\mathrm{H}), 6.4-7.02(4 \mathrm{H}, \mathrm{Ar}-\mathrm{H}), 2.23\left(\mathrm{~s}, 6 \mathrm{H}, \mathrm{CH}_{3}\right) ;{ }^{13} \mathrm{C} \mathrm{NMR}\left(\mathrm{CDCl}_{3}\right)$ $\delta=20.4,40.3,61.7,113.3,120.2,124.4,125.7,126.5,128.1,128.6,130.3$, 135.6, 136.8, 137.3, 139.9, 142.8, 146.3, 163.8; IR (KBr): $1586(\mathrm{C}=\mathrm{C}), 1643$ $(\mathrm{C}=\mathrm{N}) \mathrm{cm}^{-1}$; LC-MS (Scinpor ESI m/z): $326.34\left(\mathrm{M}^{+}\right), 327.23(\mathrm{M}+1,26.13 \%)$
MF: $\mathrm{C}_{23} \mathrm{H}_{22} \mathrm{~N}_{2}$.

3.2.8 Compound (5h): Synthesis of 2,3-dihydro-2,4-bis(4-methoxyphenyl)$1 \mathrm{H}$-benzo[b][1,5] diazepine

Yield: 95\%, MP: $112-114^{\circ} \mathrm{C}$; ${ }^{1} \mathrm{HNMR}\left(400 \mathrm{MHz}, \mathrm{CDCl}_{3}, \delta\right.$ ppm): $\delta 2.06$ (d, 2H, $\left.\mathrm{CH}_{2}\right), 3.11(\mathrm{t}, 1 \mathrm{H}, \mathrm{CH}), 4.05(\mathrm{~s}, 1 \mathrm{H}, \mathrm{NH}), 6.66-7.22(4 \mathrm{H}, \mathrm{Ar}-\mathrm{H}), 6.76-$ $7.62(4 \mathrm{H}, \mathrm{Ar}-\mathrm{H}), 6.4-7.02(4 \mathrm{H}, \mathrm{Ar}-\mathrm{H}), 3.67\left(\mathrm{~s}, 6 \mathrm{H}, \mathrm{OCH}_{3}\right) ;{ }^{13} \mathrm{C} \mathrm{NMR}\left(\mathrm{CDCl}_{3}\right)$ $\delta=40.1,54.7,63.7,112.9,113.7,115.9,124.1,125.2,126.1,127.1,129.9$, 131.7, 140.3, 143.1, 146.7, 157.3, 161.2, 164.5; IR (KBr): $1587(\mathrm{C}=\mathrm{C}), 1639$ $(\mathrm{C}=\mathrm{N}) \mathrm{cm}^{-1}$; LC-MS (Scinpor ESI m/e): m/e $358.43\left(\mathrm{M}^{+}\right), 359.33(\mathrm{M}+1$, 25.13\%); MF: $\mathrm{C}_{23} \mathrm{H}_{22} \mathrm{~N}_{2} \mathrm{O}_{2}$.

3.2.9 Compound (5i): Synthesis of 2-(2,3-dihydro-2-phenyl-1H-benzo[b] [1,5]diazepin-4-yl) phenol

Yield: 84\%, MP: $128-129^{\circ} \mathrm{C} ;{ }^{1} \mathrm{HNMR}\left(400 \mathrm{MHz}, \mathrm{CDCl}_{3}, \delta\right.$ ppm): $\delta 1.84$ $\left(\mathrm{d}, 2 \mathrm{H}, \mathrm{CH}_{2}\right), 3.72(\mathrm{t}, 1 \mathrm{H}, \mathrm{CH}), 4.15(\mathrm{~s}, 1 \mathrm{H}, \mathrm{NH}), 7.02-7.22(5 \mathrm{H}, \mathrm{Ar}-\mathrm{H}), 6.66-$ $7.40(4 \mathrm{H}, \mathrm{Ar}-\mathrm{H}), 6.3-7.02(4 \mathrm{H}, \mathrm{Ar}-\mathrm{H}), 4.89(\mathrm{~s}, 1 \mathrm{H}, \mathrm{OH}) ;{ }^{13} \mathrm{C} \mathrm{NMR}\left(\mathrm{CDCl}_{3}\right) \delta=$ 40.6, 64.7, 113.2, 116.5, 117.6, 119.9, 123.4, 124.9, 125.5, 126.1, 127.2, 129.8, 130.7, 133.8, 139.2, 142.8, 146.7, 161.3, 164.2; IR (KBr): $3410(\mathrm{O}-\mathrm{H}$, broad), $1590(\mathrm{C}=\mathrm{C}), 1640(\mathrm{C}=\mathrm{N}) \mathrm{cm}^{-1}$; LC-MS (Scinpor ESI m/z): $314.65\left(\mathrm{M}^{+}\right)$; MF: $\mathrm{C}_{21} \mathrm{H}_{18} \mathrm{~N}_{2} \mathrm{O}$.

3.2.10 Compound (5j): Synthesis of 2-(2,3-dihydro-2-(4-methoxyphenyl)1H-benzo[b][1,5] diazepin-4-yl)phenol

Yield: $96 \%$, MP: $107-108^{\circ} \mathrm{C} ;{ }^{1} \mathrm{HNMR}\left(400 \mathrm{MHz}, \mathrm{CDCl}_{3}, \delta\right.$ ppm): $\delta 1.74$ $\left(\mathrm{d}, 2 \mathrm{H}, \mathrm{CH}_{2}\right), 3.83(\mathrm{t}, 1 \mathrm{H}, \mathrm{CH}), 4.03(\mathrm{~s}, 1 \mathrm{H}, \mathrm{NH}), 6.67-7.0(4 \mathrm{H}, \mathrm{Ar}-\mathrm{H}), 6.7-7.18$ $(4 \mathrm{H}, \mathrm{Ar}-\mathrm{H}), 6.38-7.0(4 \mathrm{H}, \mathrm{Ar}-\mathrm{H}), 5.1(\mathrm{~s}, 1 \mathrm{H}, \mathrm{OH}), 3.7\left(\mathrm{~s}, 3 \mathrm{H}, \mathrm{OCH}_{3}\right) ;{ }^{13} \mathrm{C}$ NMR $\left(\mathrm{CDCl}_{3}\right) \delta=40.7,56.5,64.2,112.9,115.7,116.5,117.6,120.2,121.1,124.9$, 125.7, 126.6, 130.8, 131.6, 132.1, 142.7, 144.9, 157.3, 161.5, 163.9; IR (KBr): $3415\left(\mathrm{O}-\mathrm{H}\right.$, broad), $1591(\mathrm{C}=\mathrm{C}), 1639(\mathrm{C}=\mathrm{N}) \mathrm{cm}^{-1}$; LC-MS (Scinpor ESI m/z): $344.23\left(\mathrm{M}^{+}\right), 345.12(\mathrm{M}+1,24.1 \%)$; MF: $\mathrm{C}_{22} \mathrm{H}_{20} \mathrm{~N}_{2} \mathrm{O}_{2}$.

Table 2 Oxalic acid catalyzed synthesis of $\mathbf{5 a - j}$.

\begin{tabular}{|c|c|c|c|c|c|}
\hline Entry & $\mathrm{R}_{2}$ & $\mathrm{R}_{1}$ & $\begin{array}{l}\text { Reaction time } \\
(\mathrm{min})\end{array}$ & Yield $(\%)^{\mathrm{a}}$ & $\begin{array}{c}\text { Melting } \\
\text { Point }\left({ }^{\circ} \mathrm{C}\right) \\
\end{array}$ \\
\hline $5 a$ & & & 150 & 93 & 136-137 \\
\hline $5 b$ & & & 160 & 87 & $110-112$ \\
\hline $5 c$ & & & 155 & 89 & 96-98 \\
\hline $5 d$ & & & 160 & 76 & $113-114$ \\
\hline $5 e$ & & & 150 & 91 & $122-123$ \\
\hline $5 f$ & & & 140 & 82 & $131-132$ \\
\hline $5 g$ & & & 135 & 92 & $130-132$ \\
\hline $5 \mathrm{~h}$ & & & 130 & 95 & $112-114$ \\
\hline $5 i$ & & & 155 & 84 & $128-129$ \\
\hline $5 j$ & & & 165 & 96 & $107-108$ \\
\hline
\end{tabular}

${ }^{\text {a }}$ Yield are of pure isolated compounds. 


\section{ACKNOWLEDGEMENTS}

The authors are thankful to the Head, Department of Chemical Technology, Dr. Babasaheb Ambedkar Marathwada University, Aurangabad 431004 (MS), India for providing the laboratory facility.

\section{REFERENCES}

1. (a) J. R. De Baun, F. M. Pallos, D. R. Baker, Chem. Abstr. 86, 5498d, (1977). (b) H. Schultz Benzodiazepines, Springer Heidelberg, Germany, 1982. (c) R. K. Smiley Comprehensive Organic Chemistry, Pergamon, UK, 1979. (d) A. R. Katritzky, C. W. Rees Comprehensive Heterocyclic Chemistry, Pergamon, UK, 1984. (e) S. Garattini, E. Mussini, L. O. Randall Benzodiazepines, Raven Press, New York, 1973.

2. (a) M. Nardia, A. Cozzaa, L. Maiuoloa, M. Oliveriob, A. Procopiob, Tet. Lett. 52, 4827, (2011). (b) J. K. Chakrabarti, T. M. Hotten, I. A. Pullar, D. J. Steggles, J. Med. Chem. 32, 2375, (1989). (c) S. Andronati, E. Semenishyna, V. Pavlovsky, Y. Simonov, S. Makan, I. Boyko, N. Burenkova, M. Gdaniec, P. Cardinael, J. P. Bouillon, A. Mazepa, Eur. J. Med. Chem. 45, 1346, (2010). (d) R. Gitto, V. Orlando, S. Quartarone, G. D. Sarro, A. D. Sarro, E. Russo, G. Ferreri, A. Chimirri, J. Med. Chem. 46, 3758, (2003).

3. L. H. Sternbach, Angew. Chem. Int. Ed. 10, 34, (1971).

4. (a) V. Merluzzi, K. D. Hargrave, M. Labadia, K. Grozinger, M. Skoog, J. C. Wu, C. K. Shih, K. Eckner, S. Attox, J. Adams, A. S. Rosenthal, R. Faanes, R. J. Eckner, R. A. Koup, J. L. Sullivan, Science. 250, 1411, (1990). (b) M. Di Braccio, G. Grossi, G. Romoa, L. Vargiu, M. Mura, M. E. Marongiu, Eur. J. Med. Chem. 36, 935, (2001).

5. (a) A. M. El-Sayed, A. Khodairy, H. Salah, H. Abdel-Ghany, Phos. Sul. Sil. Relat. Elem. 182, 711, (2007). (b) G. K. Nagaraja, V. P. Vaidya, K. S. Rai, K. M. Mahadevan, Phos. Sul. Sil. Relat. Elem. 181, 2797, (2006). (c) K. Nabih, A. Baouid, A. Hasnaoui, A. Kenz, Syn. Comm. 34, 3565, (2004). (d) K. V. V. Reddy, P. S. Rao, D. Ashok, Syn. Comm. 30, 1825, (2000).

6. R. C. Haris, J. M. Straley, US 1537757, (1968).

7. J. S. Yadav, B. V. S. Reddy, G. Satheesh, G. Srinivasulu, A. C. Kunwar, Arkivoc. iii, 221, (2005). (b) R. M. Claramunt, D. Sanz, S. Aggarwal, A. Kumar, O. Prakash, S. P. Singh, J. Elgueroc, Arkivoc. xiv, 35, (2006).

8. J. A. L. Herbert, H. Suschitzky, J. Chem. Soc. Perk. Trans. 1, 2657, (1974).

9. H. R. Morales, A. Bulbarela, R. Contreras, Heterocycles. 24, 135, (1986).

10. D. I. Jung, T. W. Choi, Y. Y. Kim, I. S. Kim, Y. M. Park, Y. G. Lee, D. H. Jung, Syn. Comm. 29, 1941, (1999).

11. R. Varala, E. Ramu, N. Sreelatha, S. R. Adapa, Synlett. 7, 1009, (2006).
12. C. W. Kuo, C. C. Wang, V. Kavala, C. F. Yao, Molecules. 13, 2313, (2008).

13. M. S. Balakrishna, B. Kaboudin, Tet. Lett. 42, 1127, (2001).

14. M. Curini, F. Epifano, M. C. Marcotullio, O. Rosati, Tet. Lett. 42, 3193, (2001).

15. G. Maitri, U. Kayal, R. Karmakar, R. N. Bhattacharya, Tet. Lett. 53, 1460, (2012).

16. G. Sabitha, G. S. K. K. Reddy, K. B. Reddy, N. M. Reddy, J. S. Yadav, Adv. Synth. Cat. 346, 921, (2004).

17. S. K. De, R. A. Gibbs, Tet. Lett. 46, 1811, (2005).

18. J. S. Yadav, B. V. S. Reddy, S. P. Kumar, K. Nagaiah, Synthesis. 3, 480, (2005).

19. B. M. Reddy, P. M. Sreekanth, P. Lakshmanan, J. Mol. Catal. A: Chem. 237, 93, (2005).

20. M. A. Pasha, V. P. Jayashankara, Heterocycles. 68, 1017, (2006).

21. R.G. Jacob, C. S. Radatz, M. B. Ridrigues, D. Alves, G. Perin, E. J. Lenardao, L. Savegnago, Hetero. Chem. 22, 180, (2011).

22. B. Kaboudin, K. Navaee, Heterocycles. 55, 1443, (2001)

23. M. Pozarentzi, J. S. Stephanatou, C. A. Tsoleridis, Tet. Lett. 43, 1755 , (2002).

24. J. S. Yadav, B. V. S. Reddy, B. Eshwaraiah, K. Anuradha, Green Chem. 4, 592, (2002)

25. D. V. Jarikote, S. A. Siddiqui, R. Rajagopal, T. Daniel, R. J. Lahoti, K. V. Srinivasan, Tet. Lett. 44, 1835, (2003).

26. S. S. Palimkar, R. J. Lahoti, K. V. Srinivasan, Green Chem. 9, 146, (2007).

27. Y. J. Jiang, J. J. Cai, J. P. Zou, W. Zhang, Tet. Lett. 51, 471, (2010).

28. a) A. B. Smith, S. J. Branca, M. A. Guaciaro, P. M. Wovkulich, A. Korn Organic Synthesis Collective Volume, Wiley, New York, 1990. (b) A. I. Meyers, M. E. Flanagan Organic Synthesis Collective Volume, Wiley, New York, 1993. (c) I. R. Politzer, A. I. Meyers Organic Synthesis Collective Volume, Wiley, New York, 1988.

29. N. Rabjan Organic Synthesis Collective Volume, Wiley, New York, 1963.

30. M. R. Apelian, A. S. Fung, G. J. Kennedy, T. F. Degan, J. Phys. Chem. 100, 16577, (1996).

31. J. N. Sangshetti, N. D. Kokare, D. B. Shinde, J. Het. Chem. 45, 1191, (2008).

32. J. N. Sangshetti, N. D. Kokare, D. B. Shinde, Synthesis. 18, 2829, (2007).

33. J. N. Sangshetti, N. D. Kokare, D. B. Shinde, Chin. J. Chem. 26, 1506, (2008).

34. J. N. Sangshetti, N. D. Kokare, D. B. Shinde, Chin. Chem. Lett. 18, 1309, (2007).

35. X. Q. Pan, J. P. Zou, Z. H. Huang, W. Zhang, Tet. Lett. 49, 5302, (2008). 